\title{
A local RBF method based on a finite collocation approach
}

\author{
D. Stevens \& H. Power \\ Department of Mechanical Materials and Manufacturing Engineering, \\ University of Nottingham, UK
}

\begin{abstract}
In this work we present an overview of the RBF finite collocation approach. The RBF-FC method is an alternative decomposition for the localisation of the radial basis function (RBF) collocation method for the solution of PDEs. In contrast to the popular finite difference approach, in which the PDE governing operator is reconstructed from simple RBF interpolants, the finite collocation formulation instead enforces all governing and boundary PDEs within the local RBF collocation systems, and assembles the field variable directly into a sparse global assembly. In this way the solution of the PDE is enforced by collocation, rather than by differencing. By including the PDE governing operator within the local collocation systems any data extracted from the RBF interpolation field naturally respects the local physics of the $\mathrm{PDE}$, including information about the local convective field. This gives rise to an "implicit upwinding" effect, which allows stable solutions to be obtained in convective-dominant scenarios when using centred stencils. The approach also allows high rates of spatial convergence to be obtained. We present here an overview of the RBF-FC formulation, demonstrating the performance of the method with benchmark examples for linear elasticity and convection-diffusion PDEs. The adaptation of the method for unsteady problems is described, including a Richardson extrapolation method to improve rates of temporal convergence. The application of the method to the capture and transport of discontinuous profiles is also examined.

Keywords: meshless, radial basis functions, RBF local, finite collocation, linear elasticity, convection-diffusion, Richardson extrapolation, shock capturing.
\end{abstract}




\section{Introduction}

The radial basis function (RBF) collocation method for the solution of PDEs, as originally described by Kansa [1,2], has been applied to a variety of fields within Engineering. RBF collocation methods are attractive due to their meshless formulation, relative ease of implementation, high convergence rates, and flexibility with regards the enforcement of arbitrary boundary conditions. However the use of globally supported basis functions leads to fully-populated collocation matrices, which become increasingly ill-conditioned and computationally expensive with increasing dataset size.

One popular approach to RBF localisation is generalised finite differencing (RBF-FD). Starting from a set of scattered nodes, an RBF collocation system is formed around each internal node, covering the solution domain. In analogy to traditional finite difference methods, the local RBF systems collocate the unknown solution value at each node within the system, with the governing PDE enforced by reconstruction in order to form a sparse global linear system which expresses the value of the governing PDE operator in terms of surrounding nodal values. For more information see for example [3-5].

The proposed finite collocation approach (RBF-FC) similarly starts from a set of overlapping RBF collocation systems. However, rather than simply collocating the field variable, as in RBF-FD methods, the PDE governing operator is enforced over the stencil interior, with the field-variable enforced around the stencil periphery, and the boundary PDE operator enforced at any domain boundaries. In this way the local collocation systems are analogous to the full-domain RBF method. Since the PDE governing operator is enforced within the local collocation systems, the global assembly is obtained by reconstructing the value of the field variable at each local system centrepoint (i.e. at each internal node of the original dataset), in terms of the value of the field variable at nodes on the stencil periphery. In this way communication between adjacent systems occurs only at the stencil boundaries, and the procedure is essentially an assembly of overlapping local boundary-value systems.

Including the PDE operator within the RBF collocation systems offers a number of benefits over a simple interpolation approach. The presence of the PDE centres within the underlying interpolation systems improves the accuracy of any data extracted, leading to significantly higher rates of spatial convergence. Moreover, the local flow of information is respected, including the local convective field, allowing convective-dominant problems to be solved on centred stencils. We refer to this effect as implicit upwinding, since the weight assigned to each node within the stencil is dynamically adjusted by the method to account for the direction and magnitude of the convective field. Additionally, we observe that the RBF-FC approach is capable of predicting partial derivatives to the same order of accuracy as the field variable itself; a feature that is particularly useful for linear elasticity problems, where the accurate prediction of stress fields is often of more interest than the prediction of displacement. 


\section{Numerical formulation}

In order to fully describe the method we review first the well-known procedure for RBF collocation with partial differential operators, before describing the localisation and assembly procedure that gives rise to the RBF-FC approach. Finally, the proposed formulation for implicit time advancement is described in terms of a Crank-Nicolson procedure with Richardson extrapolation. A more complete description of the RBF-FC formulation may be found in [6], for steady problems (including linear elasticity), or [7] for transient problems.

\subsection{Traditional RBF collocation for PDEs}

We consider the Hardy multiquadric radial basis function:

$$
\psi(r)=\left(r^{2}+c^{2}\right)^{1 / 2} .
$$

Here $c$ is the RBF shape parameter, which determines the flatness of the basis functions. In practice, the value of the shape parameter can have a significant effect on solution quality for many RBF implementations. For smooth solutions a tradeoff often exists between accuracy and numerical stability; flat basis functions (i.e. high values of $c$ ) lead to more accurate solutions at the cost of worse numerical conditioning in the resulting collocation systems. For more detail, see [9, 10].

For a general linear boundary value problem

$$
\begin{array}{ll}
L[u]=f(x) & \text { on } \Omega \\
B[u]=g(x) & \text { on } \partial \Omega,
\end{array}
$$

the full-domain RBF collocation method, as described by Kansa [1, 2], constructs the solution as:

$$
u(x)=\sum_{j=1}^{N} \alpha_{j} \psi\left(\left\|x-\xi_{j}\right\|\right),
$$

where $\xi_{j}$ are a set of functional centres located at the dataset nodes. By enforcing the linear boundary operators, equation (2) at the set of nodes $x_{i}$, the following collocation system may be obtained:

$$
\left[\begin{array}{l}
B\left[\psi_{i j}\right] \\
L\left[\psi_{i j}\right]
\end{array}\right] \alpha_{j}=\left[\begin{array}{l}
g_{i} \\
f_{i}
\end{array}\right],
$$

where $\psi_{i j}=\psi\left(\left\|x_{i}-\xi_{j}\right\|\right)$. This collocation system is fully populated and nonsymmetric. In the RBF-FC method, these collocation systems are formed over each local stencil. The placement of differential operators within the local stencils is described in the following section.

As an alternative to the above-described Kansa collocation, the Hermitian collocation approach (see [8]) may also be employed in order to form the local 
collocation systems. The Hermitian approach results in a symmetric collocation matrix with stronger non-singularity properties, at the cost of increased programming complexity. A comparison of the two methods in the context of RBF-FC is given in [6]; however, in most cases the two methods offer extremely similar performance.

\subsection{Finite collocation localisation procedure}

The RBF-FC localisation procedure forms a stencil around each internal node of the domain, enforcing the PDE governing operator at the domain interior, and the field-variable (Dirichlet) operator at the domain edges. Note that, at this stage, the value of the field variable is currently unknown, and will be obtained during the global assembly. If the stencil overlaps the domain boundary then the field variable collocation is replaced by the boundary operator for nodes that lie on the domain boundary. The PDE governing operator may also be enforced at additional auxiliary locations within the stencil, further improving the solution quality; see [6]. The stencil configuration is summarised in Figure 1.

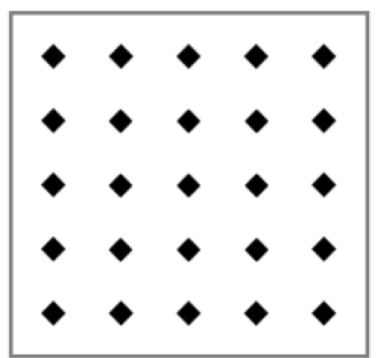

(a) RBF-FD stencil

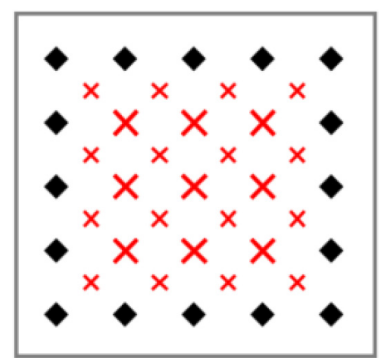

(b) RBF-FC stencil

Figure 1: Comparison of stencils for RBF finite difference (RBF-FD) and the finite collocation approach (RBF-FC). Black marks represent collocation of the unknown solution value (solution centres). Red crosses represent collocation of the PDE governing operator (PDE centres).

Having formed local RBF collocation systems around each of the $N$ internal nodes

$$
A^{(k)} \alpha^{(k)}=d^{(k)} \quad k=1, \ldots, N,
$$

the field value at any location within the support of local system $k$ may be reconstructed via equation (3). Expressing this value as a vector product we have

$$
u^{(k)}(x)=H^{(k)}(x) \alpha^{k}
$$


where $H^{(k)}(x)$ is identified as a reconstruction vector for system $k$ at location $x$. By reconstructing the field variable $u$ at the local system centrepoint, $x_{c}^{(k)}$, we obtain the following:

$$
\begin{aligned}
u^{(k)}\left(x_{c}^{(k)}\right) & =H^{(k)}\left(x_{c}^{(k)}\right) \alpha^{(k)} \\
& =H^{(k)}\left(x_{c}^{(k)}\right)\left[A^{(k)}\right]^{-1} d^{(k)} \\
& =W^{(k)}\left(x_{c}^{(k)}\right) d^{(k)} .
\end{aligned}
$$

Here $W^{(k)}\left(x_{c}^{(k)}\right)=H^{(k)}\left(x_{c}^{(k)}\right)\left[A^{(k)}\right]^{-1}$ is a stencil weights vector that expresses the value of the field variable, $u$, at the system centrepoint $x_{c}^{(k)}$, in terms of the entries in the local system data vector $d^{(k)}$. By performing the above reconstruction, equation (7), for the centrepoint of each local system, $k$, a series of $N$ simultaneous equations are formed for the $N$ unknown values of $u^{(k)}$ at the local system centrepoints. These values are obtained by solving the resulting sparse global assembly, providing the value of $u$ throughout the domain.

\subsection{Time discretisation and Richardson extrapolation}

To extend the above procedure to solve transient problems with implicit timestepping, we take a traditional finite difference of the time derivative, producing modified PDE governing operators that may be used in the local RBF collocation. In this way the transient problem is reduced to a series of inhomogeneous steadystate problems, with the inhomogeneous term storing information from the previous timestep. We consider here a Crank-Nicolson time discretisation approach along with a Richardson extrapolation to improve the rate of temporal convergence.

For a general initial boundary-value problem

$$
\begin{array}{rlll}
\frac{\partial \varphi(x, t)}{\partial t} & = & L[\varphi(x, t)]+S(x, t) & x \in \Omega \\
\varphi(x, 0) & = & f(x) & x \in \Omega \\
B[\varphi(x, t)] & = & g(x, t) & x \in \partial \Omega,
\end{array}
$$

we take a Crank-Nicolson approximation to the time derivative

$$
\frac{\varphi^{n+1}-\varphi^{n}}{\Delta t}=\frac{1}{2} L\left[\varphi^{n+1}\right]+\frac{1}{2} L\left[\varphi^{n}\right]+S(x, t),
$$

to obtain modified PDE operators 


$$
\begin{aligned}
& \bar{L}=1-1 / 2 \Delta t L \\
& \hat{L}=1+1 / 2 \Delta t L,
\end{aligned}
$$

such that

$$
\bar{L}\left[\varphi^{n+1}\right]=\hat{L}\left[\varphi^{n}\right]+S(x, t)
$$

In this way we reduce the initial boundary value problem, equation (8), to the solution of an inhomogeneous boundary problem at each timestep, with the inhomogeneous term obtained from the solution at the previous timestep.

To increase the rate of convergence in time we may implement a Richardson extrapolation. This straightforward extrapolation method, first described in [11], allows low-order terms to be eliminated from any time discretisation procedure where the rate of convergence is known. By performing a single timestep of size $h$ to obtain $\varphi_{h}$, then covering the same space with two timesteps of size $h / 2$ to obtain $\varphi_{h / 2}$, the two solutions may be combined to remove the leading order error term. For the Crank Nicolson discretisation outlined in equation (9), this increases the rate of temporal convergence from second order to fourth order. Further terms may be eliminated by considering timesteps of size $h / 4, h / 8$ etc. The extrapolation formulae for these cases and the corresponding convergence orders are outlined in Table 1.

Table 1: Richardson extrapolation formulae for second-order timestepping.

\begin{tabular}{|c|c|c|}
\hline Conv. order & Extrapolation formula & $\begin{array}{c}\text { Relative } \\
\text { computational cost }\end{array}$ \\
\hline 2 & $\varphi_{h}$ & 1 \\
\hline 4 & $\frac{4 \varphi_{h / 2}-\varphi_{h}}{4}$ & 3 \\
\hline 6 & $\frac{64 \varphi_{h / 4}-20 \varphi_{h / 2}+\varphi_{h}}{45}$ & 7 \\
\hline 8 & $\frac{4096 \varphi_{h / 8}-1344 \varphi_{h / 4}+84 \varphi_{h / 2}-\varphi_{h}}{2835}$ & 15 \\
\hline
\end{tabular}

\section{Linear elasticity results}

We consider a linear-elastic plate with a circular hole under uniform traction. This well-known benchmark problem results in a concentration of stresses around the hole (see Figure 2(b)). An analytical solution may be formulated, as given by Timoshenko and Goodier [12].

We take a Young's modulus of $210 \mathrm{GPa}$ and a Poisson ratio of 0.3 to represent mild steel, with a hole radius of $1 \mathrm{~m}$ and a far-field traction of $100 M P a$. We exploit 
the symmetry of the problem to examine a single quadrant, constraining the solution domain to a $4 \mathrm{~m} \times 4 \mathrm{~m}$ region. Over the hole we enforce the appropriate zero-traction condition, at the lines of symmetry we impose the symmetric condition (zero normal displacement and zero tangential traction), and at the farfield boundaries we enforce the analytical surface traction field, as defined by $\tau_{i}=\sigma_{i j}^{e} n_{j} . N+1$ nodes are distributed over the hole, with $N+1$ nodes in the radial direction to create a regular but non-uniform dataset (see Figure 2(a)). We consider a $5 \times 5$ stencil with auxiliary PDE centres, as shown in Figure 1(b).

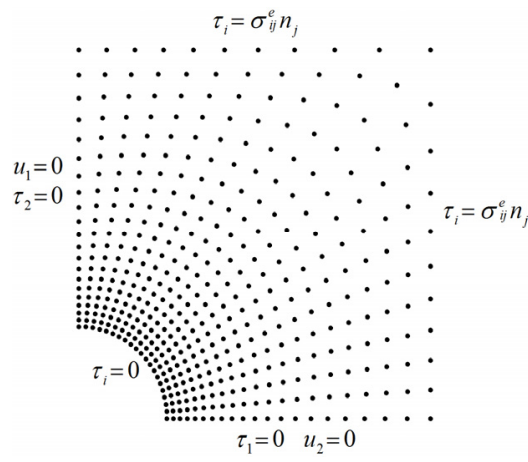

(a) Dataset $(\mathrm{N}=20)$ and $\mathrm{BCs}$

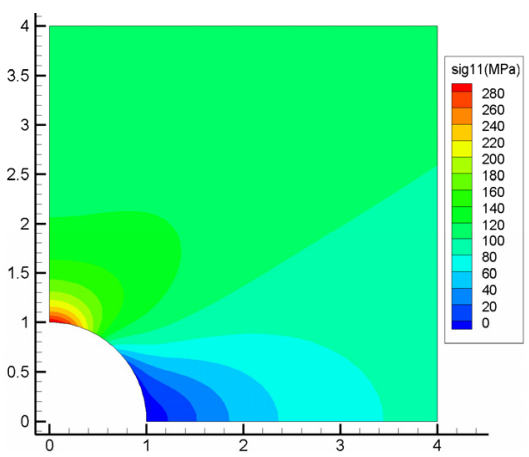

(b) Contours of stress $\left(\sigma_{11}\right)$

Figure 2: Plate with a circular hole under traction: dataset and stress contours.

Figure 3 shows the $\mathrm{L}_{2}$ relative error obtained for displacement and stresses, for datasets of size $\mathrm{N}=20,30,40,60,80$. Note that the rate of convergence for the displacement and stress fields is very similar, as is the magnitude of the errors. In FEM and similar methods, the stress fields typically exhibit significantly lower rates of convergence than does the displacement field. By contrast, the RBF-FC method is able to reproduce field-variable derivatives (such as stresses) to the same level of accuracy as the field variable itself. The rate of convergence for each solution field is around $7^{\text {th }}$ order in this case.

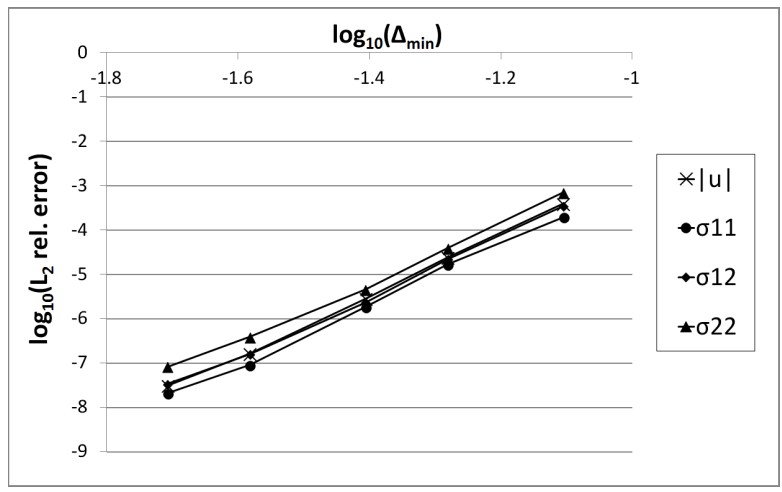

Figure 3: Spatial convergence for plate-with-a-hole case (roughly $7^{\text {th }}$ order). 


\section{Unsteady convection diffusion results}

To demonstrate the implicit upwinding effect we examine the convection of a Gaussian packet in the absence of diffusion, i.e. at infinite Péclet number:

$$
\frac{\partial \varphi}{\partial t}+u_{i} \frac{\partial \varphi}{\partial x_{i}}=0,
$$

where the Gaussian packet is defined by the initial configuration

$$
\varphi(x, 0)=e^{-200\left\|x-x_{c}\right\|} .
$$

We solve over the unit square, discretised by $N^{2}$ uniformly distributed nodes, enforcing periodic conditions at each boundary. The convective term is taken to be diagonal, i.e. $u=(1.0,1.0)$, with the profile initially located at the centre of the domain; $\varphi_{c}=(0.5,0.5)$. In this way, after time $t=1.0$ the profile performs a single diagonal cycle and returns to its initial location. The initial solution profile is shown in Figure 4.

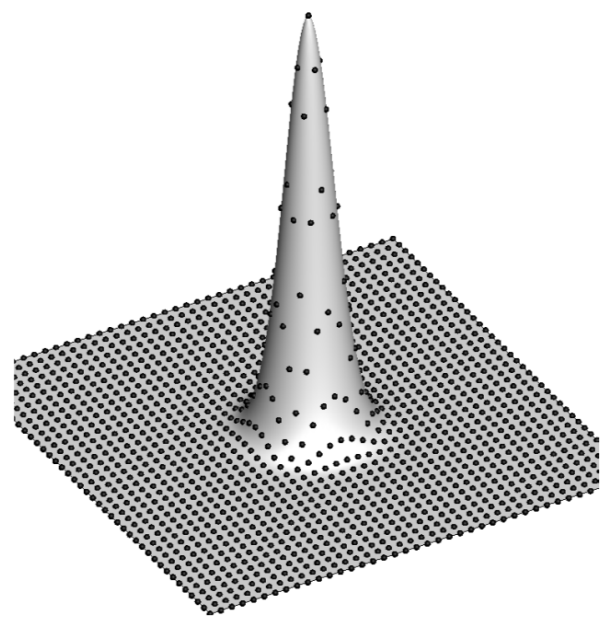

Figure 4: Initial configuration; Gaussian packet $(N=40$ dataset).

Setting the timestep size to $\Delta t=1 / N T$, we examine the solution accuracy for $N T$ ranging between 16 and 131072, with datasets of size $N=40,80,160,320$ and four different timestepping schemes; the second order Crank-Nicolson scheme without extrapolation, and Richardson extrapolation for fourth, sixth and eighthorder convergence. The stencil is a centred $5 \times 5$ patch (see Figure 1).

Figure 5 shows the results of the time convergence. The profile of each curve is consistent; the error reduces in-line with the convergence order of the timestepping scheme, until the limit of spatial accuracy is obtained. Temporal 
convergence rates are, however, somewhat restricted for large timestep sizes. The improvement from increased levels of Richardson extrapolation is clear, however the highest level of extrapolation ( $8^{\text {th }}$ order; red lines) only offers a costperformance benefit over the $6^{\text {th }}$ order scheme (green lines) at the highest dataset densities $(N=160, N=320)$. The $6^{\text {th }}$ order scheme, however, is more computationally efficient than the $2^{\text {nd }}$ and $4^{\text {th }}$ order schemes for all examined datasets. The high rate of spatial convergence can be observed from the large reduction in the small-timestep accuracy obtained by refining the dataset. Spatial convergence is roughly $8^{\text {th }}$ order in this case (see [7] for more detailed analysis).

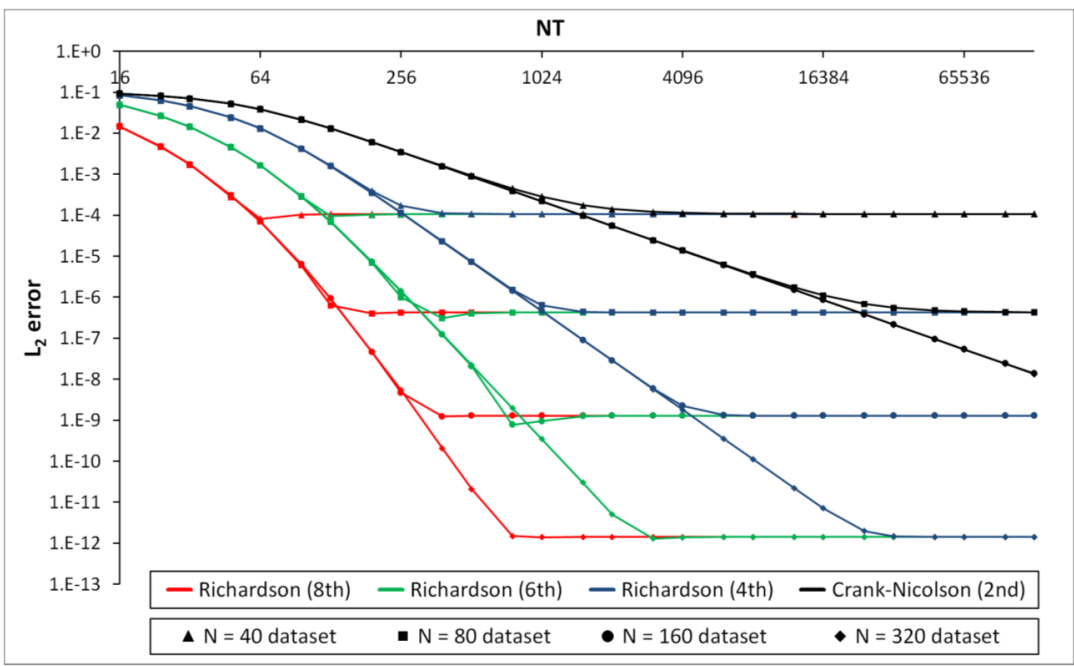

Figure 5: Gaussian packet convergence (various time-schemes and datasets).

\section{Shock capturing results}

The method is capable of capturing smooth yet strongly varying solutions in a pure-convection scenario. However, due to the smooth nature of the underlying basis functions, discontinuous profiles cannot be transported in the absence of diffusion without introducing oscillatory behaviour (see Figure 6). These oscillations are neutrally stable, i.e. they do not grow over time. By introducing a small artificial diffusion parameter, $D^{*}$ i.e. solving

$$
D^{*} \frac{\partial^{2} \varphi}{\partial x_{i}^{2}}=\frac{\partial \varphi}{\partial t} u_{i} \frac{\partial \varphi}{\partial x_{i}},
$$

the spurious oscillations may be damped, thereby stabilising the solution.

Figure 7 shows the transport of a discontinuous square-wave packet using a small, constant artificial diffusion parameter. The domain is of length 1.0, with $N$ nodes in the $x_{1}$ direction. The packet is convected for a single cycle (i.e. $t=1$ ). 
Here the value of $D^{*}$ is selected such that the magnitude of oscillations decays to below $10^{-3}$ after the cycle is completed. As can be seen in the figure, relatively little solution smearing is visible, particularly with the higher-resolution dataset (Figure 7(b)).

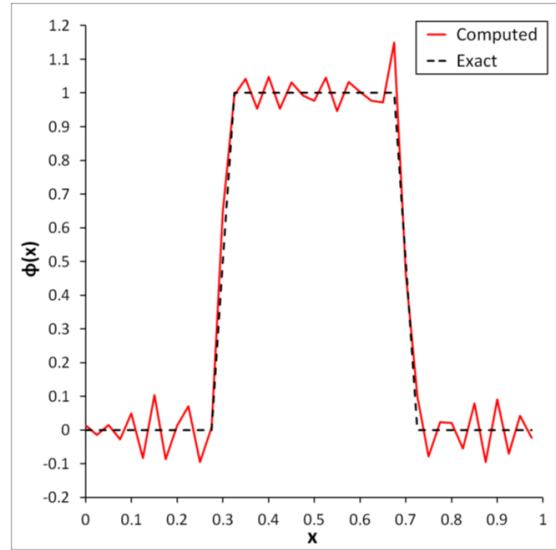

(a) $N=40$ dataset

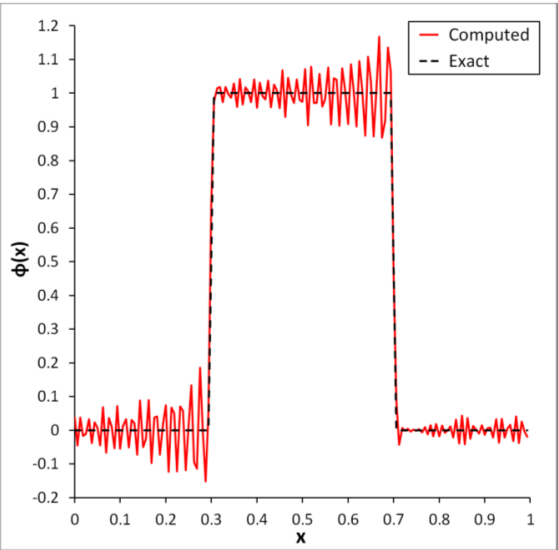

(b) $N=160$ dataset

Figure 6: Gibbs oscillations from convecting a discontinuity without smoothing.

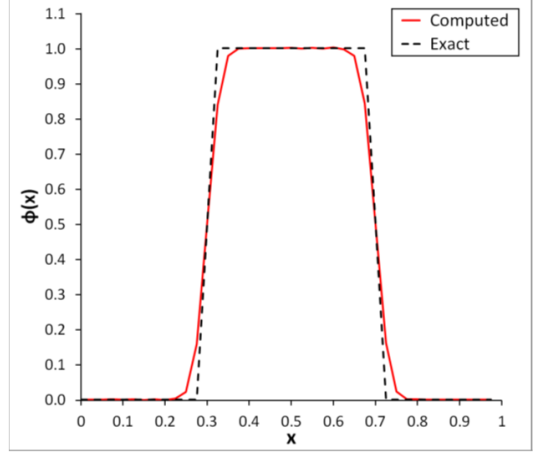

(a) $N=40$ dataset: $D^{*}=3 \times 10^{-4}$

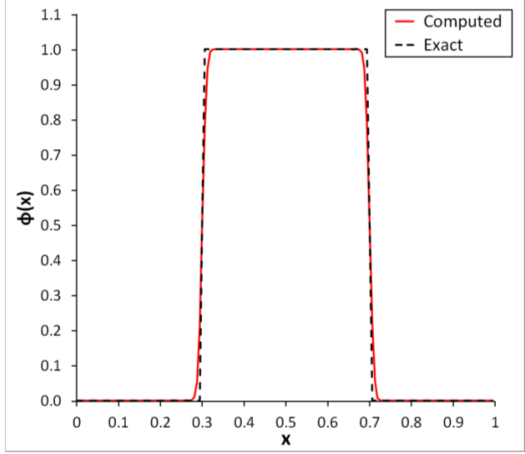

(b) $N=160$ dataset: $D^{*}=3 \times 10^{-5}$

Figure 7: Shock capturing examples using artificial diffusion.

The above implementation of the artificial stabilisation damps out oscillations steadily over time. An alternative approach is to employ the artificial diffusion in a dynamic "pulse" fashion, using larger values of $D^{*}$, but only switching on the artificial diffusion when oscillations are detected. Operating in this way the magnitude of oscillations can be constrained to within a specified tolerance 
throughout the simulation, and very long-time runs can be performed with minimal solution smearing (see [7] for more details).

\section{Summary}

We provide an overview of an alternative localisation strategy for the RBF collocation approach. The "finite collocation" formulation aims to closely resemble the structure of the global RBF collocation approach, by forming a series of local boundary value systems that enforce the governing and boundary PDE operators and communicate via their stencil boundaries. In contrast to the RBF generalised finite differencing method, the global system is obtained by assembling the field variables, rather than by reconstructing partial differential operators. In this way the solution of the PDE is driven by collocation within the RBF local systems, rather than by differencing at the global level.

This finite collocation formulation provides high convergence rates, and introduces a number of desirable numerical properties, including an "implicit upwinding" effect that allows convective-dominant solutions to be obtained on centred stencils. By exploiting the implicit upwinding feature, small amounts of artificial diffusion may be introduced to stabilise the capture of discontinuous solution profiles. The amount of artificial diffusion may be tuned to the local dataset density and desired oscillation tolerance, allowing a balance to be achieved between solution smearing and stability.

\section{References}

[1] Kansa, E. J.; Multiquadrics - A scattered data approximation scheme with applications to computational fluid-dynamics-I: Surface approximations and partial derivatives estimates, Comput. Math. Appl., 1990, 19, pp. 127145.

[2] Kansa, E. J.; Multiquadrics - A scattered data approximation scheme with applications to computational fluid dynamics-II: Solution to parabolic, hyperbolic and elliptic partial differential equations, Comput. Math. Appl., 1990, 19, pp. 147-161.

[3] Divo, E. \& Kassab, A.; An efficient localised radial basis function meshless method for fluid flow and conjugate heat transfer, Journal of Heat Transfer, 2007, 129, pp. 124-136.

[4] Flyer, N.; Lehto, E.; Blaise, S.; Wright, G. \& St-Cyr, A.; A guide to RBFgenerated finite differences for nonlinear transport: Shallow water simulations on a sphere, J. Comput. Phys., 2012, 231, pp. 4078-4095.

[5] Sarler, B. \& Vertnik, R.; Meshless explicit local radial basis function collocation methods for diffusion problems, Comput. Math. Appl., 2006, 51, pp. 1269-1282.

[6] Stevens, D.; Power, H.; Meng C. Y.; Howard, D. \& Cliffe, K.; An alternative local collocation strategy for high-convergence meshless PDE solutions, using radial basis functions, J. Comput. Phy, 2013, 254, pp. 5275 . 
[7] Stevens, D. \& Power, H.; High-Convergence Meshless Solutions to Convective-Dominant Transport Problems using Centrally Defined Stencils, J. Comput. Phys., 2014, (submitted).

[8] Wu, Z.; Hermite-Birkhoff interpolation of scattered data by radial basis functions, Approximation Theory and its Applications, 1992, 8, pp. 1-11.

[9] Kansa, E. \& Carlson, R.; Improved accuracy of multiquadric interpolation using variable shape parameters, Comput. Math. Appl., 1992, 24, pp. 99120.

[10] Wertz, J. \& Kansa, E.; The role of the multiquadric shape parameters in solving partial differential equations, Comput. Math. Appl., 2006, 51, pp. 1335-1348.

[11] Richardson, L.; The deferred approach to the limit. Phil.Trans. A, 1927, 226, pp. 299-349.

[12] Timoshenko, S. \& Goodier, J.; Theory of Elasticity, McGraw-Hill, New York, 1951. 